\title{
Instantaneous stochastic perturbation theory
}

\author{
Martin Lüscher ${ }^{a, b}$ \\ ${ }^{a}$ CERN, Physics Department, \\ Geneva 23, 1211 Switzerland \\ ${ }^{b}$ Albert Einstein Center for Fundamental Physics, Institute for Theoretical Physics, \\ Sidlerstrasse 5, Bern, 3012 Switzerland \\ E-mail: luscher@mail.cern.ch
}

ABSTRACT: A form of stochastic perturbation theory is described, where the representative stochastic fields are generated instantaneously rather than through a Markov process. The correctness of the procedure is established to all orders of the expansion and for a wide class of field theories that includes all common formulations of lattice QCD.

KEYwords: Lattice QCD, Lattice Gauge Field Theories, Lattice Quantum Field Theory, Stochastic Processes

ARXIV EPRINT: 1412.5311 


\section{Contents}

1 Introduction 2

2 Scalar field theory to second order - an illustrative example 2

2.1 Trivializing maps 3

2.2 Trivializing map to second order in the coupling 3

2.3 Breaking up the loops 4

3 Perturbation expansion to all orders $\quad 5$

$\begin{array}{ll}3.1 & \text { Abstract definition of the theory }\end{array}$

$\begin{array}{lll}3.2 & \text { Propagators and vertices } & 6\end{array}$

$\begin{array}{lll}3.3 & \text { Feynman rules } & 7\end{array}$

4 List description of Feynman diagrams $\quad 8$

4.1 Diagram data 8

4.2 Vertex and line permutations 8

$\begin{array}{ll}4.3 \text { Symmetry factors } & 9\end{array}$

5 Tree diagrams $\quad 10$

$\begin{array}{lll}5.1 \text { Random fields } & 10\end{array}$

$\begin{array}{ll}5.2 \text { Types of tree diagrams } & 11\end{array}$

6 Structure of the trivializing map $\quad 12$

$\begin{array}{lll}6.1 \text { Diagram sets } & 12\end{array}$

6.2 Ansatz for the trivializing map 12

7 Determination of the coefficients $c(\mathcal{R}) \quad 13$

$\begin{array}{lll}7.1 & \text { Disconnected parts } & 13\end{array}$

$\begin{array}{lll}7.2 & \text { Bose symmetry } & 14\end{array}$

$\begin{array}{ll}\text { 7.3 Are there more equations than unknowns? } & 14\end{array}$

$\begin{array}{ll}7.4 \text { Recursive solution of the equations } & 15\end{array}$

$\begin{array}{lll}\text { 7.5 Automated computation of the coefficients } & 16\end{array}$

8 Trivializing map in lattice QCD 16

$\begin{array}{lll}8.1 & \text { Fields, action and parameters } & 16\end{array}$

$\begin{array}{lll}\text { 8.2 Bare and renormalized perturbation expansion } & 17\end{array}$

$\begin{array}{ll}\text { 8.3 Trivializing map to second order in the coupling } & 17\end{array}$

$\begin{array}{ll}8.4 \text { Higher-order trivializing maps } & 18\end{array}$

$\begin{array}{llr}9 & \text { Concluding remarks } & 19\end{array}$ 


\section{Introduction}

Stochastic perturbation theory has its roots in stochastic quantization $[1,2]$, but is nowadays mainly used as a tool in numerical lattice field theory [3, 4] (see [5] for a review and $[6,7]$ for recent applications of the method). As in stochastic quantization, the starting point is the Langevin equation and thus a Markov process that simulates the field theory considered. The stochastic field is then expanded in a formal power series in the couplings, which allows the equation to be solved order by order in the interactions.

Numerical stochastic perturbation theory has long proved to be very useful. Often higher orders in the expansion can be reached than would be practically feasible with other methods. As the lattice spacing $a$ is taken to zero, the required numerical effort is however rapidly increasing. In four dimensions, and taking autocorrelations into account, the cost of the calculations tends to grow approximately like $a^{-6}$. Moreover, even if a higher-order scheme is used for the numerical integration of the Langevin equation [8-10], the simulation results must eventually be extrapolated to vanishing integration step size in order to be safe of uncontrolled systematic effects [11].

The form of stochastic perturbation theory described in the following sections is unrelated to stochastic quantization and is instead based on the concept of a "trivializing map" [12]. Such maps transform trivial random fields into stochastic fields representing an interacting field theory in the same way as the fields generated in a simulation do. Field configurations obtained in this way from uncorrelated random fields are statistically independent and thus provide an instantaneous simulation of the theory.

Trivializing maps practically solve the theory and cannot be expected to be easily constructible. There are, however, explicit and fairly simple maps that trivialize the theory to any finite order of perturbation theory. In the case of the one worked out in this paper, the trivializing stochastic field is given by a series of rooted tree diagrams with random fields attached to their leaves. Such diagrams can be computed through a recursive procedure, where one starts from the leaves of the diagram and progresses from one vertex to the next until the root line is reached. The trivializing field thus becomes accessible to numerical evaluation.

The principal goal in this paper is to establish the existence of trivializing maps of this kind and to provide the theoretical background for the automatic generation of the tree diagrams and the computation of their coefficients. ${ }^{1}$

\section{Scalar field theory to second order - an illustrative example}

The concepts and strategies underlying instantaneous stochastic perturbation theory are best explained in the case of a one-component scalar field $\varphi(x)$ with (Euclidean) action

$$
S(\varphi)=\int \mathrm{d}^{4} x\left\{\frac{1}{2} \partial_{\mu} \varphi(x) \partial_{\mu} \varphi(x)+\frac{1}{2} m^{2} \varphi(x)^{2}+\frac{g}{4 !} \varphi(x)^{4}\right\}
$$

\footnotetext{
${ }^{1} \mathrm{~A}$ program package providing this functionality for lattice QCD and other theories can be downloaded from http://cern.ch/luscher/ISPT.
} 
$g$ being the coupling constant and $m$ the mass parameter of the theory. An ultraviolet regularization is assumed in the following and the perturbation expansion should be the renormalized rather than the bare one, but for simplicity these complications are skipped over in this section.

\subsection{Trivializing maps}

Let $\eta(x)$ be a Gaussian random field with mean zero and variance

$$
\langle\eta(x) \eta(y)\rangle=\delta(x-y)
$$

In its simplest form, a trivializing map is a transformation of fields, where the random field $\eta(x)$ is transformed to a field $\phi(x)$ in an invertible manner such that

$$
\left\langle\phi\left(x_{1}\right) \ldots \phi\left(x_{n}\right)\right\rangle=\left\langle\varphi\left(x_{1}\right) \ldots \varphi\left(x_{n}\right)\right\rangle
$$

for all $n$ and $x_{1}, \ldots, x_{n}$. The correlation function on the right of this equation is the one determined by the action (2.1) and the associated functional integral, while on the left the product $\phi\left(x_{1}\right) \ldots \phi\left(x_{n}\right)$ is to be averaged over the Gaussian random field.

In the functional integral, trivializing maps of this kind may be considered to be transformations of integration variables. A sufficient condition for eq. (2.3) to hold is then

$$
S(\phi)-\ln \operatorname{det}\{\delta \phi / \delta \eta\}=\int \mathrm{d}^{4} x \frac{1}{2} \eta(x)^{2}+\text { constant },
$$

where $\operatorname{det}\{\delta \phi / \delta \eta\}$ denotes the Jacobian of the transformation.

A more general class of trivializing maps is obtained by allowing the trivializing stochastic field to depend on more than one random field. Since the mapping goes from several random fields to one stochastic field in this case, it cannot be considered to be a transformation of integration variables, but eq. (2.3) remains meaningful if the product of fields on the left of the equation is averaged over all random fields.

\subsection{Trivializing map to second order in the coupling}

To any finite order in the coupling, trivializing stochastic fields can be obtained in the form of a power series

$$
\phi(x)=\sum_{k=0}^{\infty} \phi_{k}(x), \quad \phi_{k}(x) \propto g^{k}
$$

with leading-order term

$$
\phi_{0}(x)=\left(-\Delta+m^{2}\right)^{-1 / 2} \eta(x) .
$$

In particular, there exist trivializing maps, where the fields $\phi_{k}(x)$ are linear combinations of Feynman diagrams of the type shown in figure 1. The first diagram, for example, is equal to

$$
-g \int \mathrm{d}^{4} y G_{0}(x, y) \phi_{0}(y)^{3}
$$




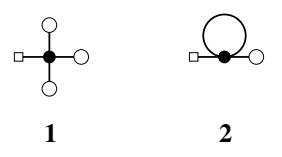

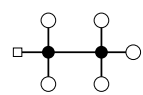

3

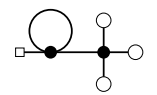

4

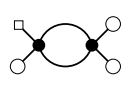

5

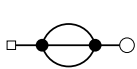

6

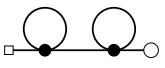

7

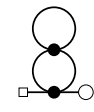

8

Figure 1. Diagrams $\mathcal{D}_{1}, \ldots, \mathcal{D}_{8}$ contributing to $\phi_{1}(x)$ (diagrams 1 and 2) and $\phi_{2}(x)$ (diagrams 3-8). The values of these diagrams are determined by the standard Feynman rules in the theory with action (2.1) except for the external lines ending in open circles, which stand for an insertion of the leading-order field (2.6). A little square is attached to the external lines that end at the point $x$.

where $G_{0}(x, y)$ denotes the free propagator in position space. Similarly, the diagram number 5 evaluates to

$$
(-g)^{2} \int \mathrm{d}^{4} y \mathrm{~d}^{4} z G_{0}(x, y) G_{0}(y, z)^{2} \phi_{0}(y) \phi_{0}(z)^{2} .
$$

No symmetry factors are associated with these diagrams and their values are calculated simply by integrating the product of the propagators, vertices and attached random fields over the positions of the vertices.

The first- and second-order terms in the expansion (2.5) of the trivializing map are then given by

$$
\begin{aligned}
\phi_{1}(x)= & \frac{1}{24} v\left(x ; \mathcal{D}_{1}\right)+\frac{1}{8} v\left(x ; \mathcal{D}_{2}\right), \\
\phi_{2}(x)= & \frac{7}{1152} v\left(x ; \mathcal{D}_{3}\right)+\frac{1}{36} v\left(x ; \mathcal{D}_{4}\right)+\frac{1}{96} v\left(x ; \mathcal{D}_{5}\right)+\frac{1}{48} v\left(x ; \mathcal{D}_{6}\right) \\
& -\frac{1}{128} v\left(x ; \mathcal{D}_{7}\right)+\frac{5}{64} v\left(x ; \mathcal{D}_{8}\right),
\end{aligned}
$$

where $v\left(x ; \mathcal{D}_{1}\right), \ldots, v\left(x ; \mathcal{D}_{8}\right)$ are the values of the diagrams drawn in figure 1 . Insertion of these expressions in eq. (2.4) shows that the map does indeed trivialize the theory up to terms of order $g^{3}$. The correctness of the coefficients in eqs. (2.9) and (2.10) can also be checked by working out the two-point, four-point and six-point correlation functions on both sides of eq. (2.3) to second order in the coupling.

\subsection{Breaking up the loops}

The trivializing map described in the previous subsection is not particularly useful, because its evaluation at order $k$ in the coupling requires the computation of Feynman diagrams with up to $k$ loops. It is possible, however, to break up the loops by introducing further Gaussian random fields

$$
\eta_{l}(x), \quad l=1,2, \ldots
$$

with mean zero and variance

$$
\left\langle\eta_{l}(x) \eta_{j}(y)\right\rangle=\delta_{l j} \delta(x-y), \quad\left\langle\eta(x) \eta_{l}(y)\right\rangle=0 .
$$




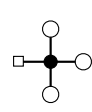

1

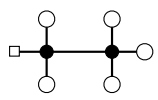

3

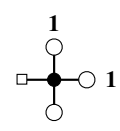

2

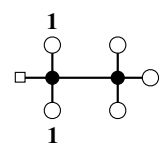

4

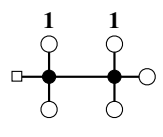

5

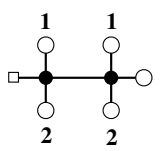

6

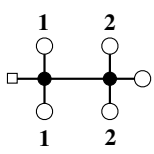

7

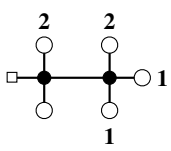

8

Figure 2. Labeled tree diagrams $\mathcal{R}_{1}, \ldots, \mathcal{R}_{8}$ corresponding to the diagrams shown in figure 1. Open circles with attached label $l$ represent the insertion of the field (2.6) with the random field $\eta$ replaced by $\eta_{l}$. Labeled open circles always come in pairs, i.e. each label occurs either zero or two times.

The contraction of the labeled random fields at the leaves of the tree diagrams shown in figure 2 then reproduces the diagrams in figure 1.

By replacing the diagrams $\mathcal{D}_{1}, \ldots, \mathcal{D}_{8}$ through the corresponding tree diagrams $\mathcal{R}_{1}, \ldots, \mathcal{R}_{8}$, the trivializing field defined through eqs. (2.6), (2.9) and (2.10) becomes a stochastic field that depends on $\eta, \eta_{1}$ and $\eta_{2}$. One might expect this field to be trivializing too, but a short calculation reveals that eq. (2.3) is violated at second order in the coupling, because some contractions of the labeled random fields generate terms that were previously not there.

As will be shown in the following sections, the trivializing property of the stochastic field can however be preserved by adjusting the coefficients of the tree diagrams. The diagram replacement and coefficient adjustment amount to the substitutions

$$
\begin{aligned}
\phi_{1}(x) \rightarrow & \frac{1}{24} v\left(x ; \mathcal{R}_{1}\right)+\frac{1}{8} v\left(x ; \mathcal{R}_{2}\right), \\
\phi_{2}(x) \rightarrow & \frac{7}{1152} v\left(x ; \mathcal{R}_{3}\right)+\frac{1}{36} v\left(x ; \mathcal{R}_{4}\right)-\frac{1}{192} v\left(x ; \mathcal{R}_{5}\right)+\frac{7}{192} v\left(x ; \mathcal{R}_{6}\right) \\
& -\frac{1}{128} v\left(x ; \mathcal{R}_{7}\right)+\frac{3}{32} v\left(x ; \mathcal{R}_{8}\right),
\end{aligned}
$$

and it is then again straightforward to check that, after these substitutions, eq. (2.3) still holds up to higher-order terms. ${ }^{2}$

\section{Perturbation expansion to all orders}

Beyond the first few orders of perturbation theory, the number of labeled tree diagrams and random-field contractions rapidly becomes very large. A mathematically precise terminology is then required to be able to establish the existence of trivializing maps of the kind discussed at the end of section 2.

\footnotetext{
${ }^{2}$ An interesting representation of the connected parts of the correlation functions $\left\langle\varphi\left(x_{1}\right) \ldots \varphi\left(x_{n}\right)\right\rangle$ is obtained by replacing all connected Feynman diagrams through the appropriate labeled tree diagrams. Such non-factorized stochastic representations are however of limited use if the observables considered are complicated functions of the fundamental fields.
} 
In this section, the class of field theories considered is specified. Furthermore, a compact notation is introduced that allows the Feynman rules to be stated economically and in full generality.

\subsection{Abstract definition of the theory}

Although instantaneous stochastic perturbation theory does not require this, a lattice regularization and a finite space-time volume are assumed from now on. For simplicity of presentation, only the case of theories containing both boson and fermion fields will be discussed. Gauge symmetries (if any) should be fixed, with ghost fields added as needed, and the boundary conditions must be such that the action has no zero modes at lowest order of perturbation theory.

The fields integrated over in the functional integral may carry various indices. It is convenient to treat the space-time coordinates as further indices and to pack all indices into a multi-index. There is then only one real boson field, $\varphi_{a}$, depending on a multi-index $a$ that includes the space-time coordinates, the Lorentz and internal-symmetry indices and an index labeling the different types boson fields.

Fermion fields (quark and ghost fields, in particular) can be similarly packed into a single field $\psi_{\alpha}$ with multi-index $\alpha$. The associated antifermion field $\bar{\psi}_{\beta}$ carries the same kind of multi-index and is taken to be independent of the fermion field (thus excluding Majorana fermions).

The total action $S$ of the theory is a function of the fields $\varphi_{a}, \psi_{\alpha}$ and $\bar{\psi}_{\beta}$, which may depend on several couplings. ${ }^{3}$ In perturbation theory, the coupling constants multiplying the interaction terms are usually taken to zero proportionally to one of them, say $g$, so that the perturbation expansion of the action

$$
S=\sum_{k=0}^{\infty} S_{k}, \quad S_{k} \propto g^{k},
$$

is an expansion in powers of $g$. At this highly abstract level, the bare and the renormalized perturbation series only differ by the parameterization of the various terms in the action.

\subsection{Propagators and vertices}

To lowest order in the coupling, the action

$$
S_{0}=\frac{1}{2} \varphi_{a} K_{a b}^{B} \varphi_{b}+\bar{\psi}_{\alpha} K_{\alpha \beta}^{F} \psi_{\beta}
$$

is assumed to be a non-degenerate quadratic expression in the fields (here and below, the Einstein summation convention is used for repeated field indices). Moreover, the kernel $K_{a b}^{B}$ must be a real symmetric matrix with strictly positive eigenvalues. The basic two-point functions are then given by

$$
\begin{gathered}
\left.\left\langle\varphi_{a} \varphi_{b}\right\rangle\right|_{g=0}=D_{a b}^{B}, \\
\left.\left\langle\psi_{\alpha} \bar{\psi}_{\beta}\right\rangle\right|_{g=0}=D_{\alpha \beta}^{F}, \quad K_{\alpha \beta}^{F} D_{b c}^{B}=\delta_{a c}^{F}=\delta_{\alpha \gamma},
\end{gathered}
$$

at this order in the coupling.

\footnotetext{
${ }^{3}$ In theories with a non-trivial functional integration measure, the action $S$ is assumed to include the terms that arise from the expansion of the measure in powers of the coupling.
} 

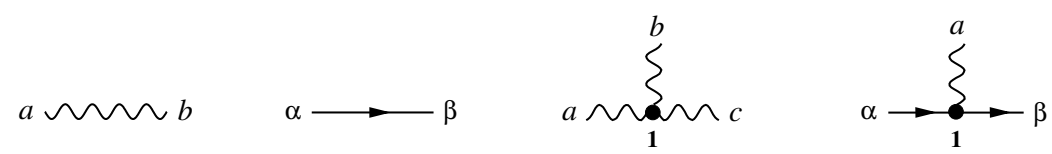

Figure 3. Graphical representation of the boson propagator $D_{a b}^{B}$, the fermion propagator $D_{\alpha \beta}^{F}$, the boson vertex $-V_{a b c}^{(1,3)}$ and the fermion-boson vertex $-W_{\alpha \beta a}^{(1,1)}$. An integer label is printed near the vertices indicating their order in the coupling (the label may be omitted if a vertex is uniquely specified by the type and number of its legs).

The interaction terms in the action are assumed to be of the general form

$$
S_{k}=\sum_{j=2}^{k+2} \frac{1}{j !} V_{a_{1} \ldots a_{j}}^{(k, j)} \varphi_{a_{1}} \ldots \varphi_{a_{j}}+\sum_{j=0}^{k} \frac{1}{j !} W_{\alpha, \beta, a_{1} \ldots a_{j}}^{(k, j)} \bar{\psi}_{\alpha} \psi_{\beta} \varphi_{a_{1}} \ldots \varphi_{a_{j}}
$$

where the vertices $V_{a_{1} \ldots a_{j}}^{(k, j)}$ and $W_{\alpha, \beta, a_{1} \ldots a_{j}}^{(k, j)}$ include the factor $g^{k}$ and are totally symmetric in the boson-field indices $a_{1}, \ldots, a_{j}$. Four-fermion interactions are not explicitly included in the action, but can be accommodated using Lagrange-multiplier fields. Clearly, many components of the propagators and vertices may vanish in a concrete case.

\subsection{Feynman rules}

The expansion of the basic correlation functions

$$
\left\langle\varphi_{a_{1}} \ldots \varphi_{a_{n}} \psi_{\alpha_{1}} \ldots \psi_{\alpha_{m}} \bar{\psi}_{\beta_{m}} \ldots \bar{\psi}_{\beta_{1}}\right\rangle
$$

in Feynman diagrams may now be derived as usual from the functional integral. The diagrams are built from the propagators and vertices introduced above, with their external lines labeled by the indices $a_{1} \ldots \beta_{m}$ of the fields in the correlation function (see figures 3 and 4). In general, the diagrams need not be connected and there may be isolated lines, i.e. lines not attached to any vertex.

The value $v(\mathcal{D})_{a_{1} \ldots \beta_{m}}$ of a diagram $\mathcal{D}$ is calculated by writing down the expressions for each graphical element (lines and vertices) and by contracting the indices of the vertices with the appropriate ones of the propagators representing the attached lines. At the other ends of external lines and the ends of the isolated lines, the indices of the propagators are set to the field indices $a_{1}, \ldots, \beta_{m}$ labeling the lines.

The contribution of the diagram to the correlation function (3.6) is equal to the product of its value, $v(\mathcal{D})_{a_{1} \ldots \beta_{m}}$, the inverse of the symmetry factor $F_{1} F_{2} F_{3}$ defined in section 4 and a fermion sign factor. Each fermion loop contributes a factor -1 to the latter and a further factor -1 is to be included if the open fermion paths in the diagram associate the fermion indices $\alpha_{1}, \ldots, \alpha_{m}$ with an odd permutation of the antifermion indices $\beta_{1}, \ldots, \beta_{m}$ (as in the second diagram in figure 4).

It may be worth noting that the diagrams considered here expand to possibly many ordinary diagrams when passing to the notation commonly used in field theory. In lattice QCD, for example, the first diagram in figure 4 includes two ordinary diagrams, one with a quark and the other with a ghost loop, both having the correct fermion-boson vertices. 

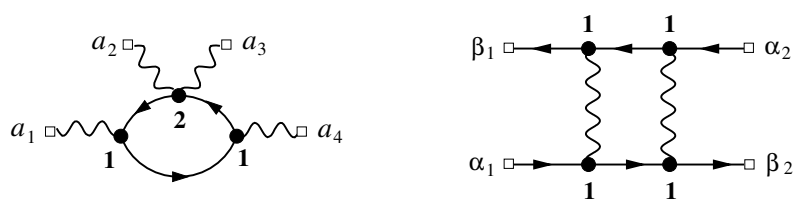

Figure 4. Two examples of diagrams complying with the rules stated in subsection 3.3. The squares at the ends of the external lines indicate that the lines are not amputated.

\section{List description of Feynman diagrams}

For the construction of trivializing stochastic fields in the most general case, a formal description of Feynman diagrams is helpful and in any case required, if the generation and structural analysis of the diagrams is to be performed by a computer.

\subsection{Diagram data}

Feynman diagrams may be described by ordered lists of vertices and lines, where the latter refer to the first in a way explained below.

Vertices are distinguished by their type (boson or fermion-boson), the order $k$ in the coupling and the number $j$ of boson legs. An example of a vertex list is

$$
\mathcal{V}=\left[V^{(1,3)}, V^{(1,3)}, V^{(2,2)}, W^{(2,0)}, W^{(2,4)}\right] .
$$

Vertex lists merely contain the vertex symbols rather than the vertices themselves. No particular ordering is required and a vertex symbol may appear more than once.

The list of lines may include internal, external and isolated lines in any order. An example of a line list is

$$
\mathcal{L}=\left[(\mathrm{b}, 1,2),(\mathrm{f}, 6,5),\left(\mathrm{f}, 5, \beta_{1}\right),\left(\mathrm{f}, \alpha_{2}, 6\right),\left(\mathrm{b}, a_{1}, a_{2}\right)\right],
$$

where the items $\left(\mathrm{b}, i_{1}, i_{2}\right)$ and $\left(\mathrm{f}, i_{1}, i_{2}\right)$ represent boson and fermion lines, respectively. The indices $i_{1}, i_{2}$ can be natural numbers or field indices. A line is outgoing at the vertex number $i_{1}$ if $i_{1}$ is a natural number and ingoing at the vertex number $i_{2}$ if $i_{2}$ is a natural number. In the case of an external line, $i_{1}$ or $i_{2}$ is the field index labeling the line (isolated lines have both indices set to field indices).

The first diagram shown in figure 4, for example, is described by the lists

$$
\begin{aligned}
& \mathcal{V}=\left[W^{(1,1)}, W^{(1,1)}, W^{(2,2)}\right] \\
& \mathcal{L}=\left[\left(\mathrm{b}, 1, a_{1}\right),\left(\mathrm{b}, 3, a_{2}\right),\left(\mathrm{b}, 3, a_{3}\right),\left(\mathrm{b}, 2, a_{4}\right),(\mathrm{f}, 1,2),(\mathrm{f}, 2,3),(\mathrm{f}, 3,1)\right] .
\end{aligned}
$$

In general, for a vertex and line list to consistently describe a diagram, the number and type of lines attached to each vertex must match the number and type of its legs. Moreover, there may be no lines attached to inexistent vertices.

\subsection{Vertex and line permutations}

The description of a diagram through a pair $(\mathcal{V}, \mathcal{L})$ of vertex and line lists implicitly goes along with a labeling of its vertices and lines. In the following, the numbers of vertices and lines will be denoted by $n_{v}$ and $n_{l}$, respectively, and the $i$ 'th vertex and $j$ 'th line are referred to as $\mathcal{V}[i]$ and $\mathcal{L}[j]$. 
There are two kinds of transformations of the diagram data $(\mathcal{V}, \mathcal{L})$, which leave the diagram unchanged:

$\left(T_{1}\right)$ The vertices may be reordered in an arbitrary way. This amounts to replacing the vertex list by a new list $\mathcal{V}^{\prime}$ such that

$$
\mathcal{V}^{\prime}[i]=\mathcal{V}[\sigma(i)] \quad \text { for all } i=1, \ldots, n_{v},
$$

where $\sigma$ is a permutation of $1, \ldots, n_{v}$. At the same time, all vertex indices $i$ in the line list must be replaced by $\sigma^{-1}(i)$.

$\left(T_{2}\right)$ The lines in the line list $\mathcal{L}$ may be reordered in an arbitrary way. In this case, the vertex list is not touched and the line list is replaced by a new list $\mathcal{L}^{\prime}$ with elements

$$
\mathcal{L}^{\prime}[j]=\mathcal{L}[\tau(j)] \quad \text { for all } \quad j=1, \ldots, n_{l},
$$

where $\tau$ is a permutation of $1, \ldots, n_{l}$. In addition, some of the boson lines may have their ends interchanged, i.e. the corresponding line items are changed according to

$$
\left(\mathrm{b}, i_{1}, i_{2}\right) \rightarrow\left(\mathrm{b}, i_{2}, i_{1}\right)
$$

The total operation then consists of a permutation of the lines and an interchange of the ends of some boson lines.

Diagram data $(\mathcal{V}, \mathcal{L})$ and $\left(\mathcal{V}^{\prime}, \mathcal{L}^{\prime}\right)$ that can be matched by applying a product of the transformations $T_{1}$ and $T_{2}$ are considered to be equivalent. By construction, there is a one-to-one correspondence between diagrams and equivalence classes of diagram data.

\subsection{Symmetry factors}

The transformations $T_{1}$ and $T_{2}$ of the diagram data generate a group of transformations with $n_{v} ! n_{l} ! 2^{n_{b}}$ elements, where $n_{b}$ denotes the number of boson lines. Some of these transformations preserve the diagram data, i.e. are such that the vertex and line lists remain unchanged. The number of elements of this subgroup of transformations is the same for all equivalent choices of the diagram data and is referred to as the symmetry factor of the diagram.

It is understood here that the field indices at the ends of the external and isolated lines are distinguished by their names. As a consequence, the transformations that preserve the diagram data cannot involve the external and isolated lines. The vertices where the external lines are attached are then also left untouched, since these are distinguished by the field indices at the ends of the attached external lines.

Given the diagram data $(\mathcal{V}, \mathcal{L})$, the symmetry factor of the diagram may therefore be calculated as the product of the following factors:

$\left(F_{1}\right)$ The number of transformations $T_{1}$ that permute the internal vertices (those without attached external lines) such that the vertex list remains the same, while the associated change of the line list can be undone by a transformation $T_{2}$. 
$\left(F_{2}\right)$ A factor $n$ ! for each instance, where two vertices are connected by $n$ (and not more than $n$ ) boson lines or where $n$ boson lines go from a vertex to itself.

$\left(F_{3}\right)$ A factor 2 for each boson line that goes from a vertex to itself.

The product of the factors $F_{2}$ and $F_{3}$ actually coincides with the number of transformations $T_{2}$ that preserve the line list.

The factors $F_{1}-F_{3}$ do not refer to the labeling of the external lines. A symmetry factor associated with the latter is

$\left(F_{4}\right)$ The number of permutations of the field indices attached to the external lines, which leave the diagram data unchanged modulo transformations $T_{1}$ and $T_{2}$.

The diagrams contributing to the correlation function (3.6), for example, divide into sets of $n !(m !)^{2} / F_{4}$ diagrams that coincide with one another except for a permutation of the field indices $a_{1}, \ldots, \beta_{m}$.

\section{Tree diagrams}

The trivializing maps constructed in the following sections are given by labeled tree diagrams like the ones shown in figure 2. In the general theory considered here, many more diagrams can be drawn and there are both boson and fermion random fields. The random fields are introduced in this section and various classes of tree diagrams are then defined.

\subsection{Random fields}

Let $\eta_{a}$ be a real-valued Gaussian random field with boson-field index $a$, mean zero and variance

$$
\left\langle\eta_{a} \eta_{b}\right\rangle=\delta_{a b}
$$

Since the boson propagator $D_{a b}^{B}$ is a real symmetric matrix with positive eigenvalues, it can be written as the square of another real symmetric matrix $M_{a b}$. The field

$$
\chi_{a}=M_{a b} \eta_{b}
$$

is then a Gaussian random field with variance

$$
\left\langle\chi_{a} \chi_{b}\right\rangle=D_{a b}^{B}
$$

and mean zero.

Random fermion and antifermion fields are treated differently and are given by

$$
\zeta_{\alpha}=D_{\alpha \beta}^{F} \rho_{\beta}, \quad \bar{\zeta}_{\beta}=\rho_{\beta}^{*},
$$

where $\rho_{\alpha}$ is a complex Gaussian random field with fermion-field index $\alpha$, mean zero and variance

$$
\left\langle\rho_{\alpha} \rho_{\beta}\right\rangle=0, \quad\left\langle\rho_{\alpha} \rho_{\beta}^{*}\right\rangle=\delta_{\alpha \beta} .
$$




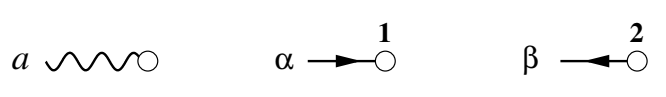

Figure 5. Random fields attached to the vertices of a diagram are represented by external lines ending in an open circle. From left to right, the random fields represented by the lines in this figure are $\chi_{a}, \zeta_{1, \alpha}$ and $\bar{\zeta}_{2, \beta}$. The labels drawn near the circles are the indices of the labeled random fields.

The two-point function

$$
\left\langle\zeta_{\alpha} \bar{\zeta}_{\beta}\right\rangle=D_{\alpha \beta}^{F}
$$

then reproduces the fermion propagator, but the fact that random fermion fields are pseudofermion fields (i.e. complex fields with fermion-field indices) should be kept in mind.

Further random boson fields $\chi_{k, a}, k=1,2, \ldots$, and random fermion fields $\zeta_{k, \alpha}$ (together with the antifermion fields $\bar{\zeta}_{k, \alpha}$ ) will be needed as well. The fields in these sequences are referred to as the labeled random fields. All random fields are assumed to be statistically independent from one another.

Random fields can be attached to the vertices of a Feynman diagram by replacing external lines through random fields of the appropriate type (see figure 5). The value of such a diagram depends on the random fields and is therefore a stochastic observable. It is calculated by substituting the expressions for the propagators, vertices and random fields for the corresponding graphical elements and by contracting the field indices at the vertices. There is no intervening propagator between the random fields and the vertices to which they are attached.

\subsection{Types of tree diagrams}

A tree diagram is a connected diagram with one or more vertices and no loops. The following types of tree diagrams with attached random fields will be considered:

(1) Unlabeled diagrams. All external lines of these tree diagrams represent one of the random fields $\chi_{a}, \zeta_{\alpha}$ or $\bar{\zeta}_{\beta}$ and are therefore unlabeled.

(2) Labeled diagrams. These diagrams are obtained by replacing some of the random fields at the leaves of an unlabeled tree diagram through labeled random fields. The latter must come in pairs with matching type and index. Moreover, each pair may occur at most once and the ends of each fermion path in the diagram must be assigned the same label or remain both unlabeled.

(3) Rooted labeled diagrams. The diagrams of this kind are labeled tree diagrams, where one unlabeled external boson line is replaced by an ordinary external boson line (i.e. by a line representing the boson propagator).

Labeled diagrams need not have any labeled external lines, i.e. the unlabeled tree diagrams are included in the set of labeled diagrams. An example of each type of diagram is shown in figure 6. 

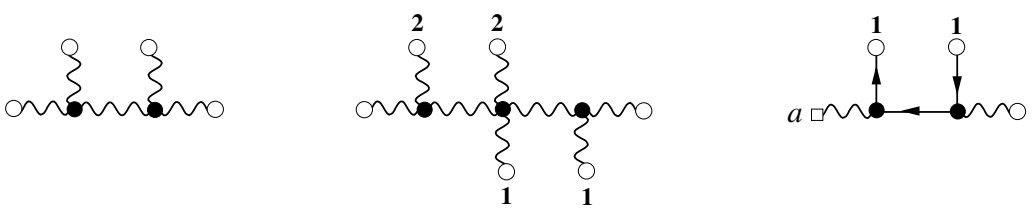

Figure 6. Examples of unlabeled, labeled and rooted labeled tree diagrams. Random-field labels must come in pairs, but boson and fermion labels may be the same, since they refer to different sequences of random fields. For simplicity, the vertex labels are omitted in this figure.

\section{$6 \quad$ Structure of the trivializing map}

The strategy in the following is to write down a structured ansatz for the trivializing stochastic field and to show that the trivializing property, eq. (2.3), is guaranteed, if the adjustable parameters are chosen appropriately.

\subsection{Diagram sets}

The construction starts by introducing certain sets

$$
L_{k, l}, \quad k=1,2, \ldots, \quad l=0,1, \ldots, \bar{l}(k),
$$

of labeled tree diagrams, where $k$ is the order in the coupling and $l$ the number of labeled pairs of external lines of the diagrams in $L_{k, l}$. Since tree diagrams of order $k$ have at most $k+2$ external lines, the maximal number $\bar{l}(k)$ of labeled pairs cannot be larger than $\lfloor k / 2\rfloor+1$.

$L_{k, 0}$ coincides with the set of unlabeled tree diagrams of order $k$. For $l \geq 1$ the sets $L_{k, l}$ are obtained by going through the diagrams in $L_{k, 0}$ and labeling $l$ pairs of external lines in all possible ways. For each type of field (boson or fermion), the pairs are labeled from 1 in steps of 1 . Diagrams that coincide after contracting the labeled external lines are not distinguished and only one of them is to be included in $L_{k, l}$.

Another family of diagram sets, $R_{k, l}$, derives from the sets $L_{k, l}$ by selecting the diagrams with at least one unlabeled external boson line and no unlabeled external fermion lines. Each of these diagrams is then transformed to a rooted labeled tree diagram by replacing an unlabeled external boson line through an ordinary external boson line. For notational convenience, the set $R_{0,0}$, whose only element is the first diagram in figure 5 , is included in this family of sets.

The construction of the sets $R_{k, l}$ of diagrams depends on some arbitrary choices to be made in the step leading from $L_{k, 0}$ to $L_{k, l}$ and when the root line is selected. There are, in principle, alternative constructions, where the diagrams in $R_{k, l}$ are replaced by linear combinations diagrams, but the number of diagrams contributing to the trivializing field is then likely to increase.

\subsection{Ansatz for the trivializing map}

As in numerical lattice QCD, the fermion fields $\psi_{\alpha}$ and $\bar{\psi}_{\beta}$ are integrated out and the primary correlation functions considered are those of the boson field $\varphi_{a}$. The trivializing 
stochastic field $\phi_{a}$ is then a boson field too, but must take the effects of the fermion loops into account so that the correlation functions of the full theory are properly reproduced.

Following the lines of section 2, the field $\phi_{a}$ is expanded in a series

$$
\begin{aligned}
\phi_{a} & =\sum_{k=0}^{\infty} \phi_{k, a} \\
\phi_{k, a} & =\sum_{l=0}^{\bar{l}(k)} \sum_{\mathcal{R} \in R_{k, l}} c(\mathcal{R}) v(\mathcal{R})_{a},
\end{aligned}
$$

where $v(\mathcal{R})_{a}$ denotes the value of the diagram $\mathcal{R}$ with field index $a$ at the end of the root line. The coefficient $c(\mathcal{R})$ of the leading term,

$$
\phi_{0, a}=\chi_{a}
$$

is set to unity, but the coefficients of the tree diagrams are left unspecified at this point. With increasing order in the coupling, the trivializing field depends on more and more random fields. Their number however grows only linearly with the maximal number of loops Feynman diagrams can have at the order considered.

The ansatz (6.3) and the way in which the fermion random fields were introduced in general leads to complex trivializing fields. In purely bosonic theories, the field is real if (and only if) the action has no imaginary part. Complex trivializing fields do not give rise to any conceptual difficulties in perturbation theory, since the expectation values of all observables are eventually expressed through the basic correlation functions of the boson field and these are correctly reproduced by the trivializing field.

\section{Determination of the coefficients $c(\mathcal{R})$}

The coefficients of the tree diagrams in the expansion (6.3) of the trivializing stochastic field are to be chosen such that

$$
\left\langle\phi_{a_{1}} \ldots \phi_{a_{n}}\right\rangle=\left\langle\varphi_{a_{1}} \ldots \varphi_{a_{n}}\right\rangle
$$

for all $n \geq 1$ and to all orders in the coupling. At any given order, both correlation

functions in eq. (7.1) evaluate to sums of ordinary diagrams, the ones on the left being obtained by Wick-contracting the random fields on which the stochastic fields $\phi_{a_{1}}, \ldots, \phi_{a_{n}}$ depend. The equation thus holds algebraically if the diagrams and their coefficients are the same on the left and the right.

\subsection{Disconnected parts}

The connected and disconnected parts of the correlation functions are related to each other through the moment-cumulant transformation and thus in a way that does not refer to any particular properties of the correlation functions. Equation (7.1) is therefore satisfied if and only if the connected parts of the correlation functions are the same. 
Since tree diagrams are connected, the connected parts of the correlation functions of the stochastic field coincide with the sum of the connected diagrams obtained by the Wick contraction of the random fields. As a consequence, it suffices to ensure that the connected diagrams and their coefficients are the same on the two sides of eq. (7.1) for the equation to be fully satisfied.

\subsection{Bose symmetry}

The connected diagrams contributing to the correlation function $\left\langle\varphi_{a_{1}} \ldots \varphi_{a_{n}}\right\rangle$ divide into subsets of diagrams that coincide with one another up to a permutation of the field indices $a_{1}, \ldots, a_{n}$. In general, there are fewer than $n$ ! diagrams in a subset, but it always contains all diagrams with different index assignments. Since the diagrams have the same symmetry and sign factors, the sum of their contributions is totally symmetric in the field indices and given by

$$
\frac{(-1)^{n_{\mathrm{F}}}}{F_{1} F_{2} F_{3} F_{4}} \sum_{\pi \in \mathcal{S}_{n}} v(\mathcal{D})_{a_{\pi(1)} \ldots a_{\pi(n)}},
$$

where $\mathcal{D}$ is any diagram in the subset, $n_{\mathrm{F}}$ the number of fermion loops in the diagram and $\mathcal{S}_{n}$ the group of permutations of $1, \ldots, n$. The product of the symmetry factors $F_{1} \ldots F_{4}$ includes $F_{4}$ in order to correct for the fact that not all index permutations yield different diagrams (cf. subsection 4.3).

Practically the same remarks apply in the case of the diagrams generated by the Wick contractions on the left of eq. (7.1). Since the stochastic field is bosonic, its correlation functions may be written in the manifestly symmetric form

$$
\left\langle\phi_{a_{1}} \ldots \phi_{a_{n}}\right\rangle=\sum_{\mathcal{R}_{1} \in R} \ldots \sum_{\mathcal{R}_{n} \in R} c\left(\mathcal{R}_{1}\right) \ldots c\left(\mathcal{R}_{n}\right) \frac{1}{n !} \sum_{\pi \in \mathcal{S}_{n}}\left\langle v\left(\mathcal{R}_{1}\right)_{a_{\pi(1)}} \ldots v\left(\mathcal{R}_{n}\right)_{a_{\pi(n)}}\right\rangle .
$$

The sums in this equation run over the union $R$ of the sets $R_{k, l}$ of diagrams. They are formal sums that only make sense at any finite order in the coupling.

The contractions of the random fields on the right of eq. (7.3) produce terms proportional to

$$
\sum_{\pi \in \mathcal{S}_{n}} v(\mathcal{D})_{a_{\pi(1)} \ldots a_{\pi(n)}}
$$

where $\mathcal{D}$ is a Feynman diagram with $n$ ordinary boson external lines (and no further external lines). Matching the two sides of eq. (7.1) thus amounts to matching the coefficients of these index-symmetrized diagram values.

\subsection{Are there more equations than unknowns?}

The discussion in the previous subsections shows that there is one coefficient equation per connected diagram $\mathcal{D}$ with $n \geq 1$ ordinary external boson lines, where diagrams related by a permutation of the field indices $a_{1}, \ldots, a_{n}$ labeling the external lines are not distinguished. In the following, the notation $[\mathcal{D}]$ is used for diagrams $\mathcal{D}$ modulo field-index permutations.

The ansatz for the trivializing stochastic field, on the other hand, depends on one adjustable coefficient $c(\mathcal{R})$ per rooted labeled tree diagram $\mathcal{R} \in R_{k, l}$. By construction, 
each diagram $\mathcal{R}$ derives from a unique labeled tree diagram in $L_{k, l}$, which in turn maps to an ordinary Feynman diagram $[\mathcal{D}]$ when its labeled external lines are contracted and the unlabeled ones are replaced by ordinary external lines. This relation between $\mathcal{R}$ and $[\mathcal{D}]$ is one-to-one and such that

$$
\sum_{\pi \in \mathcal{S}_{n}}\left\langle v(\mathcal{R})_{a_{\pi(1)}} \phi_{0, a_{\pi(2)}} \ldots \phi_{0, a_{\pi(n)}}\right\rangle=(n-1) ! \sum_{\pi \in \mathcal{S}_{n}} v(\mathcal{D})_{a_{\pi(1)} \ldots a_{\pi(n)}}+\ldots,
$$

where the ellipsis stands for the contributions of the disconnected diagrams produced by the contractions of the random fields on the left of the equation. Moreover, all diagrams $[\mathcal{D}]$ are obtained from a diagram $\mathcal{R}$ in this way.

At any given order in the coupling, there are therefore exactly as many equations as there are unknown coefficients.

\subsection{Recursive solution of the equations}

The equations for the coefficients $c(\mathcal{R})$ may now be solved one after the other in a particular order. As explained in the previous subsections, there is one coefficient equation per connected ordinary diagram $[\mathcal{D}]$ with right-hand side equal to

$$
\frac{(-1)^{n_{\mathrm{F}}}}{F_{1} F_{2} F_{3} F_{4}} \text {. }
$$

On the left of the equation, there is a sum of terms that are obtained by evaluating the expectation values in eq. (7.3). More precisely, the term contributed by a given combination $\mathcal{R}_{1}, \ldots, \mathcal{R}_{n}$ of rooted labeled tree diagrams is given by

$$
c\left(\mathcal{R}_{1}\right) \ldots c\left(\mathcal{R}_{n}\right) \frac{N_{\mathrm{c}}}{n !},
$$

with $N_{\mathrm{c}}$ being the number of Wick contractions of the random fields in the product $v\left(\mathcal{R}_{1}\right)_{a_{1}} \ldots v\left(\mathcal{R}_{n}\right)_{a_{n}}$ which yield the diagram $[\mathcal{D}]$.

There are two types of diagram combinations $\mathcal{R}_{1}, \ldots, \mathcal{R}_{n}$ that can contract to the diagram $[\mathcal{D}]$ :

(1) Two or more of the diagrams are tree diagrams and are thus contained in some of the sets $R_{k, l}$ with $k \geq 1$. The order in the coupling of all diagrams $\mathcal{R}_{1}, \ldots, \mathcal{R}_{n}$ must then be strictly less than the one of $\mathcal{D}$.

(2) Only one of the diagrams is non-trivial, say $\mathcal{R}_{1} \in R_{k, l}$ for some $k \geq 1$ and $l \geq 0$. The order $k$ of the diagram must then be the same as the one of $\mathcal{D}$. Moreover, the number $l$ of labeled pairs of external lines cannot exceed the number of loops of $\mathcal{D}$. If $l$ coincides with the number of loops, the construction of the sets $R_{k, l}$ of diagrams implies that $\mathcal{R}_{1}$ must be equal to $\mathcal{R}$, the rooted labeled tree diagram associated with $[\mathcal{D}]$.

The equations for the coefficients can therefore be solved recursively with increasing order $k$ in the coupling and, at fixed $k$, increasing loop number $l$. For a given diagram $[\mathcal{D}]$, the equation is of the form

$$
c(\mathcal{R})+\text { already known terms }=\frac{(-1)^{n_{\mathrm{F}}}}{F_{1} F_{2} F_{3} F_{4}}
$$


and can therefore be solved for the coefficient $c(\mathcal{R})$ of the tree diagram $\mathcal{R}$ associated with $[\mathcal{D}]$.

\subsection{Automated computation of the coefficients}

The number of tree diagrams contributing to the trivializing stochastic field at order $k$ increases roughly like $k$ !. An automated computation of their coefficients is therefore desirable and can follow essentially the steps described in this section.

First the various sets of diagrams must be generated. It is advisable to introduce a partial ordering of the diagrams based on their structure and to build up binary search trees [13] together with the sets. The computer time spent to find the diagrams generated by the contractions of the random fields on the right of eq. (7.3) otherwise grows with the square of the number of diagrams.

The diagram ordering also allows the number of terms contributing to the sum over diagrams in eq. (7.3) to be reduced by a large factor by summing over ordered combinations $\mathcal{R}_{1}, \ldots, \mathcal{R}_{n}$ of diagrams only. Another acceleration of the calculations can be achieved by collecting contractions of random fields, which only differ by a permutation of some vertex legs of equal type and therefore obviously yield the same contracted diagram.

\section{Trivializing map in lattice QCD}

The abstract theory considered in the previous sections includes all popular formulations of lattice QCD as special cases, provided the chosen boundary conditions in finite volume are such that the gauge modes are the only zero modes of the action. This property is guaranteed with Schrödinger-functional (SF) [14, 15] and open-SF boundary conditions [16], for example. The aim in the present section is to describe and further develop the trivializing map in lattice QCD, using a slightly less abstract language.

\subsection{Fields, action and parameters}

In perturbation theory, the gauge-field variables $U(x, \mu) \in \mathrm{SU}(3)$ may be represented through a real-valued gauge potential $A_{\mu}^{a}(x)$ according to

$$
U(x, \mu)=\exp \left\{g_{0} A_{\mu}^{a}(x) T^{a}\right\},
$$

where $g_{0}$ denotes the bare gauge coupling and $T^{a}, a=1, \ldots, 8$, a basis of anti-Hermitian $\mathrm{SU}(3)$ generators (for notational convenience, the lattice spacing is set to unity). The gauge degrees of freedom then need to be fixed by adding a gauge-fixing term and the associated Faddeev-Popov ghosts to the theory. Usually a lattice version of the covariant gauge with bare gauge parameter $\lambda_{0}$ is chosen.

Further parameters of the lattice theory are the number $N_{\mathrm{f}}$ of quark flavours, the quark masses and optionally a set of irrelevant parameters, the so-called improvement coefficients, which are tuned so as to reduce the effects of the non-zero lattice spacing on the calculated physical quantities. For simplicity, the dependence of the improvement coefficients on the gauge coupling is however ignored in the following and the quarks are assumed to be mass-degenerate with bare mass parameter $m_{0}$. 


\subsection{Bare and renormalized perturbation expansion}

The bare perturbation expansion of the correlation functions of the gauge potential is obtained by inserting the representation (8.1) of the link variables in the action and by expanding the functional integral in powers of $g_{0}$. In addition to the gluon vertices that derive from the gauge action, the expansion of the integration measure gives rise to further vertices of order $g_{0}^{l}$ with $l=2,4, \ldots$ gluon legs. There are quark-gluon vertices with any number of gluon legs and ghost-gluon vertices with $1,2,4, \ldots$ gluon legs (see ref. [17], for example, for all-order formulae for the measure and ghost-gluon vertices).

The renormalization of the gauge coupling and the gauge potential merely amounts to a reordering of the bare perturbation expansion, but the renormalization of the gaugefixing parameter and the quark mass requires additional diagrams with gauge-fixing and mass vertex insertions to be calculated. This complication can economically be dealt with by substituting

$$
\lambda_{0}=\lambda+\delta \lambda, \quad m_{0}=m+\delta m,
$$

for the bare gauge-fixing and mass parameters, where $\lambda$ and $m$ are held fixed, while the terms proportional to $\delta \lambda$ and $\delta m$ in the action are treated as perturbations of order $g_{0}^{2}$. The exact form of the associated vertices depends on the chosen lattice action, but they normally have only two legs.

\subsection{Trivializing map to second order in the coupling}

The rooted labeled tree diagrams contributing to the stochastic gauge potential that trivializes the lattice theory to a given order of perturbation theory are made of the vertices mentioned in subsection 8.2 and the gluon, quark and ghost propagators. In order to minimize the number of diagrams and still be able to easily pass to the renormalized expansion, the best strategy is to expand the field in the bare coupling and to include the gauge-fixing and quark-mass vertices in the Feynman rules.

Random quark fields must normally carry a flavour index, but for mass-degenerate quarks, trivializing gauge potentials can be constructed with flavour-less random fields and coefficients $c(\mathcal{R})$ that depend on $N_{\mathrm{f}}$. The first- and second-order terms in the expansion of the trivializing field are then given by

$$
\begin{aligned}
\mathcal{A}_{1}= & \frac{1}{6} v\left(\mathcal{R}_{1}\right), \\
\mathcal{A}_{2}= & \frac{1}{2} v\left(\mathcal{R}_{2}\right)+\frac{1}{2} v\left(\mathcal{R}_{3}\right)+\frac{1}{24} v\left(\mathcal{R}_{4}\right)+\frac{1}{8} v\left(\mathcal{R}_{5}\right)-\frac{1}{2} v\left(\mathcal{R}_{6}\right)-\frac{1}{2} N_{\mathrm{f}} v\left(\mathcal{R}_{7}\right) \\
& +\frac{5}{72} v\left(\mathcal{R}_{8}\right)+\frac{1}{12} v\left(\mathcal{R}_{9}\right)-\frac{1}{2} v\left(\mathcal{R}_{10}\right)-\frac{1}{2} N_{\mathrm{f}} v\left(\mathcal{R}_{11}\right),
\end{aligned}
$$

where, for brevity, the coordinates and indices $x, \mu, a$ have been suppressed. The diagrams $\mathcal{R}_{1}, \ldots, \mathcal{R}_{11}$ are shown in figure 7 . There are no diagrams $\mathcal{R}$ in this list, where the associated contracted diagram $[\mathcal{D}]$ has tadpole subdiagrams, ${ }^{4}$ since $v(\mathcal{D})_{a_{1} \ldots a_{n}}=0$ in this case and the

\footnotetext{
${ }^{4} \mathrm{~A}$ tadpole subdiagram is a subdiagram without external lines, which is connected to the rest of the diagram through a single gluon line.
} 


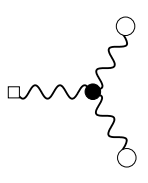

1

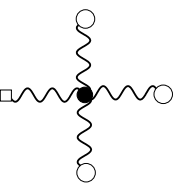

4

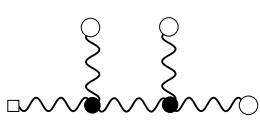

8

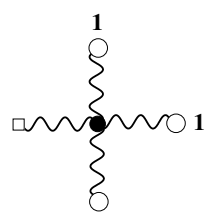

5

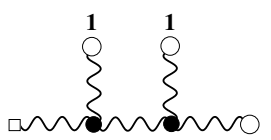

9

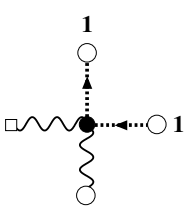

6

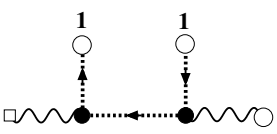

10

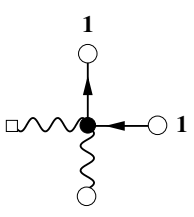

7

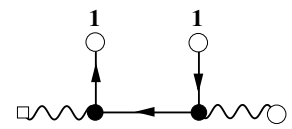

11

Figure 7. Rooted labeled tree diagrams $\mathcal{R}_{1}, \ldots, \mathcal{R}_{11}$ contributing to the trivializing gauge potential at first order in the gauge coupling (diagram 1) and at second order (diagrams 2-11). Shaded square vertex symbols represent the vertices that derive from the gauge-field integration measure and shaded circular symbols stand for the insertion of a gauge-fixing vertex proportional to $\delta \lambda$. Quark and ghost lines (solid and dotted) are distinguished in these diagrams.

associated coefficient equation consequently does not need to be satisfied. The coefficient $c(\mathcal{R})$ may therefore be set to zero (cf. section 7 ).

\subsection{Higher-order trivializing maps}

Apart from the growing combinatorial complexity, the construction of the trivializing gauge potential at higher orders in the coupling does not run into additional difficulties. Quarkmass vertices and tree diagrams with more than one branch first appear at fourth order. At order $k$, the coefficients of the tree diagrams are polynomials in $N_{\mathrm{f}}$ of degree up to $\lfloor k / 2\rfloor$ with rational coefficients.

In view of the large number of diagrams, the generation of the diagram sets $R_{k, l}$ and the calculation of the coefficients of the diagrams rapidly becomes a task that calls for automation. As explained in subsection 8.3, the tree diagrams that contract to diagrams with tadpole subdiagrams can be omitted from the beginning. The other tree diagrams can be generated following the lines of subsection 6.1. Some arbitrary choices need to be made at this point, but the numbers of diagrams quoted in the second and forth column of table 1 do not depend on these.

Even with a straightforward selection rule like the one used to produce the figures in table 1, surprisingly many diagrams turn out to have vanishing coefficient (see $3 \mathrm{rd}$ column of table 1). If the diagrams with gauge-fixing and ghost vertices are omitted, the numbers 


\begin{tabular}{|c|rr|rr|}
\hline order & all & $c(\mathcal{R}) \neq 0$ & no ghosts & $c(\mathcal{R}) \neq 0$ \\
\hline 1 & 1 & 1 & 1 & 1 \\
2 & 10 & 10 & 7 & 7 \\
3 & 19 & 19 & 14 & 14 \\
4 & 146 & 141 & 91 & 87 \\
5 & 522 & 489 & 309 & 286 \\
6 & 4042 & 3524 & 2106 & 1816 \\
7 & 20312 & 16851 & 9978 & 8274 \\
8 & 159190 & 127143 & 70854 & 56986 \\
\hline
\end{tabular}

Table 1. Numbers of rooted labeled tree diagrams $\mathcal{R}$ in lattice QCD.

of diagrams are reduced by about a factor 2 (columns 4 and 5). These diagrams can actually be dropped in the case of open-SF boundary conditions [16], for example, if the temporal gauge $A_{0}^{a}(x)=0$ is chosen.

\section{Concluding remarks}

The viability of the trivializing fields constructed in this paper for numerical stochastic perturbation theory still needs to be demonstrated. As already mentioned, rooted labeled tree diagrams can be evaluated through a recursive procedure that starts from the leaves of the diagrams and visits the vertices one after another until the root line is reached. At each vertex the attached fields and branches are combined to form a new field, which is then propagated to the next vertex. Highly efficient algorithms (the fast Fourier transform [18] and multigrid methods [19]) are available for the field propagation, which amounts to solving a free-field equation with known right-hand side.

The rapid growth of the number of diagrams with the order in the coupling potentially sets a sharp limit on the applicability of instantaneous stochastic perturbation theory. There are, however, various ways to organize the computations efficiently, exploiting the product structure of tree diagrams. Reusing already calculated branches, for example, or factoring out the vertices recursively, starting from the root line, may result in significant speed-up factors and thus allow the calculations to be driven to higher orders than appears to be practically feasible at first sight.

An intriguing aspect of instantaneous stochastic perturbation theory is the fact that theories with complex action are not excluded and do not even require a special treatment. In particular, chiral gauge theories [20] and QCD at finite baryon density are among the accessible theories.

Open Access. This article is distributed under the terms of the Creative Commons Attribution License (CC-BY 4.0), which permits any use, distribution and reproduction in any medium, provided the original author(s) and source are credited. 


\section{References}

[1] G. Parisi, Y.-S. Wu, Perturbation theory without gauge fixing, Sci. Sin. 24 (1981) 483 [INSPIRE].

[2] P.H. Damgaard and H. Hüffel, Stochastic quantization, Phys. Rept. 152 (1987) 227 [INSPIRE].

[3] F. Di Renzo, G. Marchesini, P. Marenzoni and E. Onofri, Lattice perturbation theory on the computer, Nucl. Phys. Proc. Suppl. 34 (1994) 795 [InSPIRE].

[4] F. Di Renzo, E. Onofri, G. Marchesini and P. Marenzoni, Four loop result in SU(3) lattice gauge theory by a stochastic method: Lattice correction to the condensate, Nucl. Phys. B 426 (1994) 675 [hep-lat/9405019] [INSPIRE].

[5] F. Di Renzo and L. Scorzato, Numerical stochastic perturbation theory for full QCD, JHEP 10 (2004) 073 [hep-lat/0410010] [INSPIRE].

[6] M. Brambilla, M. Dalla Brida, F. Di Renzo, D. Hesse and S. Sint, Numerical stochastic perturbation theory in the Schrödinger functional, PoS(Lattice 2013) 325 [arXiv: 1310.8536] [INSPIRE].

[7] M. Dalla Brida and D. Hesse, Numerical stochastic perturbation theory and the gradient flow, PoS(Lattice 2013) 326 [arXiv: 1311.3936] [INSPIRE].

[8] G.G. Batrouni, G.R. Katz, A.S. Kronfeld, G.P. Lepage, B. Svetitsky and K.G. Wilson, Langevin simulations of lattice field theories, Phys. Rev. D 32 (1985) 2736 [INSPIRE].

[9] A. Ukawa and M. Fukugita, Langevin simulation including dynamical quark loops, Phys. Rev. Lett. 55 (1985) 1854 [INSPIRE].

[10] G.S. Bali, C. Bauer, A. Pineda and C. Torrero, Perturbative expansion of the energy of static sources at large orders in four-dimensional SU(3) gauge theory, Phys. Rev. D 87 (2013) 094517 [arXiv: 1303.3279] [INSPIRE].

[11] A.S. Kronfeld, Dynamics of Langevin simulations, Prog. Theor. Phys. Suppl. 111 (1993) 293 [hep-lat/9205008] [INSPIRE].

[12] M. Lüscher, Trivializing maps, the Wilson flow and the HMC algorithm, Commun. Math. Phys. 293 (2010) 899 [arXiv:0907.5491] [INSPIRE].

[13] D.E. Knuth, The art of computer programming. Volume 3. Sorting and serching, Addison-Wesley (1973).

[14] M. Lüscher, R. Narayanan, P. Weisz and U. Wolff, The Schrödinger functional - a renormalizable probe for non-Abelian gauge theories, Nucl. Phys. B 384 (1992) 168 [hep-lat/9207009] [INSPIRE].

[15] S. Sint, On the Schrödinger functional in QCD, Nucl. Phys. B 421 (1994) 135 [hep-lat/9312079] [INSPIRE].

[16] M. Lüscher, Step scaling and the Yang-Mills gradient flow, JHEP 06 (2014) 105 [arXiv: 1404.5930] [INSPIRE].

[17] M. Lüscher, Selected topics in lattice field theory, in E. Brézin and J. Zinn-Justin, Fields, strings, critical phenomena: proceedings, North-Holland, Amsterdam The Netherlands (1990), [Conf. Proc. C 880628 (1988) 451] [inSPIRE]. 
[18] C. van Loan, Computational frameworks for the fast Fourier transform, Frontiers in applied mathematics (Book 10), Society for Industrial and Applied Mathematics, Philadelphia U.S.A. (1992).

[19] Y. Saad, Iterative methods for sparse linear systems, 2nd ed., Society for Industrial and Applied Mathematics, Philadelphia U.S.A. (2003) and online at http://www-users.cs.umn.edu/ saad/.

[20] M. Lüscher, Lattice regularization of chiral gauge theories to all orders of perturbation theory, JHEP 06 (2000) 028 [hep-lat/0006014] [INSPIRE]. 